\title{
Electromagnetic radiation in multiply connected Robertson-Walker cosmologies
}

\author{
Roman Tomaschitz \\ Dipartimento di Matematica Pura ed Applicata dell' Università degli Studi di Padova, \\ Via Belzoni 7, I-35131 Padova, Italy and Center for Nonlinear Studies, \\ Los Alamos National Laboratory, MS-B258, Los Alamos, New Mexico 87545
}

(Received 11 November 1992; accepted for publication 26 March 1993)

\begin{abstract}
Maxwell's equations on a topologically nontrivial cosmological background are studied. The cosmology is locally determined by a Robertson-Walker line element, but the spacelike slices are open hyperbolic manifolds, whose topology and geometry may vary in time. In this context the spectral resolution of Maxwell's equations in terms of horospherical elementary waves generated at infinity of hyperbolic space is given. The wave fronts are orthogonal to bundles of unstable geodesic rays, and the eikonal of geometric optics appears just as the phase of the horospherical waves. This fact is used to attach to the unstable geodesic rays a quantum mechanical momentum. In doing so the quantized energy-momentum tensor of the radiation field is constructed in a geometrically and dynamically transparent way, without appealing to the intricacies of the second quantization. In particular Planck's radiation formula, and the bearing of the multiply connected topology on the fluctuations in the temperature of the background radiation is discussed.
\end{abstract}

\section{INTRODUCTION}

This is the third part of a series ${ }^{1,2}$ of papers on the global topological and geometrical structure of space-time, and its implications on classical and quantum mechanics. We are studying the global behavior of world lines, rays, particles, fields, classical or quantized, in an infinite space that has a nontrivial topological structure. And we figure out common features that do indeed exist between the classical and quantum description of objects moving in this space, which is itself a dynamical object, for its topology and geometry may vary in time.

Though contemporary cosmology originated from general relativity, the input that comes from this theory is rather small and qualitative: the existence of a space-time line element, the geodesic principle, the principle of general covariance. Additional assumptions are needed to fix the actual form of the line element: the principles of homogeneity and isotropy assure that the three-space is of constant curvature at any given instant of time. That determines the line element apart from a time-dependent expansion factor $a(\tau)$ that sets the length scale in the three-space, and apart of the sign $k(k=-1,0$, or +1$)$ of the curvature of the three-space. The line element can then be cast into the form $d s^{2}=-c^{2} d \tau^{2}+4 a^{2}(\tau)\left(1+k|\mathbf{x}|^{2} / R^{2}\right)^{-2} d x^{2}$. That is just the Minkowski metric with Euclidean three-space replaced by the $a(\tau)$-scaled, constantly curved three-space. Such line elements are usually referred to as of Robertson-Walker ( RW) type.

What have Einstein's equations then to contribute to cosmology? Clearly they do not determine the metric, even not locally, because for that we would have to know the energymomentum tensor of the universe, which is not the case. If we insert the RW metric into the Einstein equations we can however express the energy and the pressure density of the universe as a function of $a(\tau)$ and its derivatives. The requirement of positivity of energy and pressure gives then restrictions on the asymptotic behavior of $a(\tau)$, for example, in the case $k=-1$ positivity would require $a(\tau) \sim \tau$ for $\tau \rightarrow \infty$. In the limit $\tau \rightarrow 0$ (or $\tau \rightarrow-\infty$, if one believes in an infinite past) positivity arguments of this kind are not reliable, because in this limit either 
pressure or energy density diverge to infinity, and the classical Einstein equations are not supposed to give even qualitatively correct results in this regime. Anyhow, the expansion factor, the pressure, and the energy density remain unknown functions, and even if one postulates $a d$ hoc and in addition a universal thermodynamic equation of state this situation is not remedied. I do not want to deny here the considerable heuristic and phenomenological value of such supplements, however we are here more interested in the underlying structure of spacetime, and on its bearing on the microscopic dynamics, and about that these equations have little to say.

Up to now we have only addressed the local, metric structure, the line element. In cosmology a much more important question is the global structure of space-time, the global topology, which is clearly left totally undecided by general relativity, being a purely metrical theory. Usually this question is settled in textbooks by postulating maximal symmetry, the existence of a continuous six-parameter symmetry group transitively acting on the spacelike slices. This postulate restricts then the possible topologies to be either Euclidean space $(k=0)$, or the three-sphere $(k=1)$, or projective three-space $(k=1)$, or a shell of the Minkowski hyperboloid $(k=-1)$. Moreover there is only one metric of constant curvature that one can impose on these topologies. Maximal symmetry implies perfect homogeneity and isotropy.

This principle of maximal symmetry of the three-space is of course very convenient, because it fixes once and for all both the topology and the geometry. However we regard it as too rigid, too static, unnecessary restrictive, and we suggest to relax it by requiring instead of maximal symmetry constant (negative) curvature. Moreover we assume that the three-space is infinite. Then the topology does not any more determine the metric uniquely, which therefore may itself vary in time, apart from the scaling with the expansion factor. Locally, on sufficiently small coordinate patches we have still the six-parameter group of isometries, and the space is locally homogeneous and isotropic. For a more pictorial description of isotropy, homogeneity, and symmetry in this context we refer to Ref. 3. Finally I mention that as soon as the topology is nontrivial there does not any more exist a continuous group of global isometries, in a sense it is replaced by the discrete covering group of the three-space manifold.

Because the actual impact of general relativity is rather small, one has to pose the question in how far Riemannian geometry enters at all in cosmologies with constantly curved spaces. Though it provides the underlying structure of the principle of general relativity, the actual use of Riemannian geometry is trivialized by the simplicity of the RW line element. At first, the time coordinate in the line element is extremely distinguished, which highly encourages the use of three-dimensional formalism, using time as a parameter. The second reason is that the constant sectional Gaussian curvature of the three-space makes the use of the Riemann curvature tensor superfluous, and renders the three-space more to an object of elementary geometry. This is perhaps the main reason that Newtonian theories of cosmology still enjoy a certain popularity.

The paper is organized as follows. In Secs. II and III the spectral theory of Maxwell's equations in infinite, multiply connected spaces is on the agenda. The spectrum and the eigenfunctions are explicitly calculated, and the orthogonality and completeness relations are derived. Many of the technicalities used in the spectral theory of the scalar wave equations in Refs. 1 and 2 have been employed here without further mentioning, likewise most of the notation. In particular this holds true for Sec. II, where the spectral theory in the Poincare upper half-space $H^{3}$, isometric to the Minkowski hyperboloid, is developed. In Sec. IV nothing new happens, but $I$ hope it enhances a little the understanding of electromagnetism in $R W$ cosmologies. Maxwell's equations and conservation laws in terms of $\mathbf{E}$ and $\mathbf{B}$ fields on the spacelike slices are formulated.

In Sec. V we discuss the Planck radiation formula for multiply connected RW cosmologies, 
employing the close relation between wave mechanics and the unstable classical mechanics in RW cosmologies. ${ }^{1,2}$ In the case of the electromagnetic field it happens that the eikonal of ray optics appears as the phase in the elementary waves that we identified in Secs. II and III as the eigenfunctions. This provides an exact and concrete geometric content of the Einstein formula $p_{\mu}=\hbar k_{\mu}$, which we use to attach to the rays a definitive quantum mechanical momentum. This enables us then to construct the quantized energy-momentum tensor in a painless and unambiguous way. Finally we close Sec. VI with some comments on the recently discovered fluctuations in the microwave background, and demonstrate how they fit into the cosmic scene advocated here. For further discussion we refer to the Conclusion, Sec. VI.

\section{THE GENERAL SETTING: MAXWELL's EQUATIONS IN THE POINCARÉ HALF- SPACE $\boldsymbol{H}^{\beta}$}

To avoid the use of unduly heavy Riemannian geometry like three-indices and the Ricci tensor, it is convenient to adopt the following form of Maxwell's equations,

$$
\begin{gathered}
\frac{\partial}{\partial x^{\kappa}}\left[\sqrt{-g} g^{\lambda v} g^{\kappa \mu}\left(\frac{\partial A_{\mu}}{\partial x^{\nu}}-\frac{\partial A_{v}}{\partial x^{\mu}}\right)\right]=0, \\
\frac{\partial}{\partial x^{\lambda}}\left(\sqrt{-g} g^{\lambda v} A_{v}\right)=0, \quad A_{0}=0 .
\end{gathered}
$$

We will use only coordinate transformations that are time-independent, and the Coulomb gauge (2.2) is covariant with respect to them. Most of the notation used here concerning hyperbolic geometry has been defined in Refs. 1 and 2, which are the prerequisite for this paper, otherwise it is self-contained. Greek indices run from 0 to 3, Latin from 1 to 3 . We write $x^{\mu}=(\tau, x)$, very often we will use less conventional complex notation, $x^{\mu}=(\tau, z, t), z=y_{1}+i y_{2}$, always $g_{00}=-c^{2}, g_{i k}=h^{2} a^{2}(\tau) \delta_{i k}, g_{0 i}=0$. For $h$; that specifies the three-space metric, we will use mostly $h=R / t$ in the Poincaré half-space model $H^{3}$, or $h=2 /\left(1-|x|^{2} / R^{2}\right)$ in the $B^{3}$ model, cf. Refs. 4 and 5.

Because of the special form of the metric $g_{\mu \nu}$, and because of Eq. (2.2), the zero component of the system (2.1) is identically satisfied. In (2.1) and (2.2) we make a variable separation, $A_{i}=\varphi(\tau) \hat{A}_{i}(\mathbf{x})$, with the separation constant $\lambda^{2} / R^{2}$, and obtain

$$
\begin{gathered}
\frac{\lambda^{2}}{R^{2}} \hat{A}_{i}=\frac{1}{h} \frac{\partial}{\partial x^{k}}\left[\frac{1}{h}\left(\frac{\partial \hat{A}_{i}}{\partial x^{k}}-\frac{\partial \hat{A}_{k}}{\partial x^{i}}\right)\right], \\
\frac{\partial}{\partial x^{k}}\left(h \hat{A}_{k}\right)=0, \quad \varphi^{ \pm}(s, \tau)=\exp \left(\mp i s \Lambda \int_{\tau_{0}}^{\tau} a^{-1}(\tau) d \tau\right),
\end{gathered}
$$

the $\varphi^{ \pm}$constitute the two fundamental solutions of the equation for the time component, and we have put $\lambda=-i s, \Lambda:=c / R$.

Next we solve Eqs. (2.3) and (2.4) in the $H^{3}$ model with $h=R / t$. The generalized eigenfunctions of the scalar wave equation ${ }^{1,2}$ are powers $P^{\lambda}$ of the Poisson kernel

$$
P(z, t, \xi)=\frac{t R}{\left(|z-\xi|^{2}+t^{2}\right)} .
$$

The $\lambda, \xi \in \mathbb{C}$ are spectral variables.

The wave fronts, $P^{\lambda}(z, t, \xi)=$ const, with $\lambda, \xi$ fixed, are horospheres. ${ }^{2}$ If $\xi=\infty$, we can take $P^{\lambda}(\xi=\infty)=(t / R)^{\lambda}$. In this case the wave fronts are just Euclidean planes in $H^{3}$, parallel to the boundary $\mathbb{C}$. For further details we refer to the Appendix of Ref. 2. 
pp. $3136-48$ in attached file 
Clearly the $h^{i j}$ on the manifold $F$ must be automorphic, $h^{i j}(x)=\left[\gamma^{\prime} x\right]_{n}^{-1 i}\left[\gamma^{\prime} x\right]_{m}^{-1 j} h^{n m}(\gamma x)$, with respect to $\Gamma$, compare Eq. (3.1). So we can require for $\chi$ the same transformation rule as for $\psi$, namely, $\chi(\gamma x, \eta, \tau)=\chi\left(x, \gamma^{-1} \eta, \tau\right)+f(\eta), f$ being independent of $(x, \tau)$. Finally, if we integrate $\widetilde{T}^{\Gamma}{ }_{\mu \nu}$, the periodized $\widetilde{T}_{\mu \nu}$, over $\cup f_{k}$ we arrive at

$$
\widetilde{T}_{\alpha \beta}^{\Gamma}(x, v) d v \sim \frac{2 c}{h R^{2}} \int_{|\eta|=R} p_{\alpha} p_{\beta}(x, \eta, \tau, v)\left[\exp \left(\frac{h v}{k \widetilde{T}(x, \eta, \tau)}\right)-1\right]^{-1} P^{2}(x, \eta) d \Omega(\eta) v d v,
$$

compare Eqs. (5.16)-(5.18).

Then there arises the question how to construct explicitly symmetric automorphic tensor fields $h^{i j}$, so that $g^{i j}+\epsilon h^{i j}$ gives curvature $-a^{-2}(\tau) R^{-2}$. It is easy to see that the dimension of this deformation space is finite-dimensional, depending on the topology. The construction of such fields is closely related to the construction of quasiconformal deformations $s^{5,8}$ of $\Gamma$. Let $F$ be the fundamental polyhedron, and $\gamma_{i}$ the generators of $\Gamma$ that provide the face identification. Let us assume now that the $\gamma_{i}$ are time-dependent, but so that the relations among them (if there are any) are preserved. Accordingly, the fundamental polyhedron $F(\tau)$ varies too. If $F\left(\tau_{0}\right)$ and $F\left(\tau_{1}\right)$ are two polyhedra that are not congruent, (i.e., cannot be mapped onto each other by a Möbius transformation), then the Poincare metric induced from the covering space $H^{3}$ turns them into two globally nonisometric manifolds-but both have the same constant sectional curvature.

That is not yet that what we need, because the transformations $x \rightarrow \gamma_{i} x$ do not leave the RW line element invariant, since $\gamma_{i}$ is time-dependent. However there exists a quasiconformal diffeomorphism $q_{\tau}$ of $H^{3}$ onto itself, so that $q_{\tau}(F(\tau))=F\left(\tau_{0}\right)$ and $q_{\tau} \Gamma(\tau) q_{\tau}^{-1}=\Gamma\left(\tau_{0}\right)$, compare Sec. 4 of Ref. 8, where such deformations have been constructed on the boundary of $H^{3}$. With this $q_{\tau}$ we can transport the metric of $F(\tau)$ onto $F\left(\tau_{0}\right)$, i.e., $q_{\tau}$ applied to the Poincare metric on $H^{3}$ gives a tensor field $\widetilde{g}_{i j}(x, \tau):=\left[q_{\tau}^{-1} x\right]_{i}^{m}\left[q_{\tau}^{-1} x\right]_{j}^{n} g_{m n}\left(q_{\tau}^{-1}(x), \tau\right)$ that is automorphic with respect to $\Gamma\left(\tau_{0}\right), \widetilde{g}_{i j}(x, \tau)=\left[\gamma_{\tau_{0}}^{\prime} x\right]_{i}^{m}\left[\gamma_{\tau_{0}}^{\prime} x\right]_{j}^{n} \widetilde{g}_{m n}\left(\gamma_{\tau_{0}}(x), \tau\right)$, for all $\gamma_{\tau_{0}} \in \Gamma\left(\tau_{0}\right)$. The metric $\widetilde{g}_{i j}$ on $F\left(\tau_{0}\right)$ is time-dependent, but both $F\left(\tau_{0}\right)$ and $\Gamma\left(\tau_{0}\right)$ are time-independent, and thus the extended RW line element $d \tilde{s}^{2}:=-c^{2} d \tau^{2}+\widetilde{g}_{i j}(x, \tau) d x^{i} d x^{j}$ is invariant with respect to $\Gamma\left(\tau_{0}\right)$.

\section{CONCLUSION AND OUTLOOK}

In this conclusion we reflect a little about wave optics, ray optics, and quantization in the cosmological models treated here. In Secs. II and III we realized that an electromagnetic wave traveling through space-time is a purely geometric-topological object, determined only by the RW line element and the topology of the space-time manifold. (The topology is imprinted on the solutions by imposing periodic boundary conditions on the fundamental polyhedron, i.e., periodicity with respect to the covering group $\Gamma$.) In the Maxwell equations there are no additional adjustable parameters involved, in the metric there are two, the speed of light and the curvature radius $R$. In simply connected spaces like the Minkowski hyperboloid there does not exist a natural length unit, in multiply connected spaces we may take for $R$ the diameter of the domain of chaoticity, ${ }^{3,7} C(\Lambda) \backslash \Gamma$, in the three-space manifold.

We constructed the spectral resolution of Maxwell's equations in terms of a complete set of elementary waves generated at some point ( $\eta$ or $\xi$ in Secs. II and III) at infinity of the three-space manifold. These elementary waves are infinitely extended objects and have no direct physical meaning, but superposing them one obtains wave packets of a finite size and one can attach to them an energy as indicated in Sec. IV. This energy is in turn a purely geometric number, cf. Eq. (4.11). Moreover these elementary waves match nicely with geometric ray optics in the cosmological background under consideration. That comes as follows. 
Though the eikonal equation may be regarded as the massless limit of the Hamilton-Jacobi equation, it turns out that ray optics gives a rather pale image of classical mechanics. That is so because one cannot define the concept of momentum in the massless limit without introducing a new fundamental constant. In fact it would not be worthwhile to consider ray optics here at all, were it not that in these cosmologies appropriate bundles of such rays, which are by the way unstable and expanding, are just the orthogonal trajectories to the wave fronts of the elementary waves, cf. Sec. V, and Ref. 2. This clearly suggests to define the momentum proportional to the wave vector $k_{\mu}$, and Planck's constant and Einstein's relation is that what we need to attach to the rays a momentum $\hbar k_{\mu}$. Having identified the photon momenta with the horospherical wave vectors, it is a straightforward exercise to construct with them the energy-momentum tensor on the three-space manifold, cf. Eqs. (5.9) and (5.16).

There are three ingredients in Planck's radiation formula (5.1), the density of eigenmodes, their frequency, and finally the distribution function of the eigenmodes. Quantum mechanics enters two places, via the Einstein relation, attaching to a frequency an energy, and perhaps in the choice of the distribution function. ${ }^{15}$ A derivation of this formula from 'first principles' I do not know. But I would like to mention here that the popular interpretation of this formula, namely, representing the field as harmonic oscillators hanging on a lattice in Fourier space cannot be retained, because a corresponding dual hyperbolic lattice in the multiply connected three-space can never exist. Hyperbolic lattices with finite cells are rigid and cannot be expanded into the thermodynamic limit.

A possibility to derive Planck's formula in this context is perhaps to consider in real three-space horospherical bundles of rays. As mentioned the wave fronts are always orthogonal to them, and the classical (Lyapunov) instability of the rays makes these bundles to statistical objects. One could try to do statistics with them instead of the energy of oscillators in momentum space.

\section{ACKNOWLEDGMENTS}

The author acknowledges the support of the European Communities through their Science Program under Grant No. B/SC1*-915078. Part of this work was performed under the auspices of the US Department of Energy.

\footnotetext{
${ }^{1}$ R. Tomaschitz, J. Math. Phys. 32, 2571 (1991).

${ }^{2}$ R. Tomaschitz, J. Math. Phys. 34, 1022 (1993).

${ }^{3}$ R. Tomaschitz, Foundations of Classical and Quantum Chaos in Extended RW-cosmologies, to appear in the Proceedings of the XIX International Colloquium on Group Theoretical Methods in Physics, Salamanca, Spain, 1992, edited by J. Mateos-Guilarte et al. (CIEMAT, Madrid).

${ }^{4}$ A. Beardon, The Geometry of Discrete Groups (Springer, Berlin, 1983).

${ }^{5}$ L. V. Ahlfors, Möbius Transformations in Several Dimensions, Lecture Notes, University of Minnesota, 1981.

${ }^{6}$ E. M. Lifshitz and I. M. Khalatnikov, Adv. Phys. 12, 185 (1963).

${ }^{7}$ R. Tomaschitz, Int. J. Theoret. Phys. 31, 187 (1992).

${ }^{8}$ R. Tomaschitz, Complex Systems 6, 137 (1992).

${ }^{9}$ D. A. Hejhal, Memoirs of the AMS 88, No. 437 (1990).

${ }^{10}$ N. Mandouvalos, Proc. London Math. Soc. 57, 209 (1988).

${ }^{11}$ S. J. Patterson, in Analytical and Geometrical Aspects of Hyperbolic Space, edited by D. B. A. Epstein, London Math. Soc. Lecture Notes 111 (Cambridge University, London, 1987), pp. 281-323.

${ }^{12}$ R. Tomaschitz, Physica D 34, 42 (1989).

${ }^{13}$ L. D. Landau and E. M. Lifshitz, The Classical Theory of Fields (Pergamon, London, 1971).

${ }^{14}$ R. Tomaschitz, in Chaotic Dynamics: Theory and Practice, edited by T. Bountis, NATO-ASI Series (Plenum, New York, 1992).

${ }^{15}$ J. H. Jeans, The Dynamical Theory of Gases (Cambridge University, Cambridge, 1921).

${ }^{16}$ C. W. Misner, K. S. Thorne, and J. A. Wheeler, Gravitation (Freeman, New York, 1973).

${ }^{17}$ S. Weinberg, Gravitation and Cosmology (Wiley, New York, 1972).

${ }^{18}$ G. F. Smoot et al., Astrophys. J. 396, L1 (1992).

${ }^{19}$ R. K. Sachs and A. M. Wolfe, Astrophys. J. 147, 73 (1967).
} 